\title{
Una rinnovata rivista scientifica delle dipendenze
}

A partire da questo numero, il periodico trimestrale delle Federazione Italiana degli Operatori dei Dipartimenti e dei Servizi e delle Dipendenze si rinnova a livello sostantivo e contenutistico.

Obiettivo è l'indicizzazione sui repertori internazionali allo scopo di aumentare visibilità e autorevolezza della rivista anche fuori dal nostro paese.

Un passaggio importante e necessario per una pubblicazione che ha fatto proprio e rappresentato il patrimonio di esperienze accumulato nella storia più che trentennale dei Servizi per le Dipendenze in Italia, interpretando la visione di un concetto complesso dell'addiction e del suo trattamento, con la sua dimensione biologica, individuale e sociale.

Si tratta di un cambiamento coerente con la filosofia operativa di una Società Scientifica come FeDerSerD, che, da sempre attenta alle premesse epistemiche alla base dello sviluppo dei fenomeni di consumo patologico e del loro trattamento, offre regolarmente ai professionisti delle dipendenze nuove occasioni di conoscenza, aggiornamento e confronto.

Si cerca di affrontare l'impegno e la responsabilità di sostenere e migliorare un settore della sanità e del welfare del nostro paese, sapendo che non solo la capacità di agire, ma anche di decidere e di comprendere, rischiano di essere compromesse dalla riduzione delle risorse e dall'impoverimento di un pensiero teso soltanto alla sopravvivenza.

Per evitare che ciò accada si chiede quindi attenzione non solo alla politica, ma soprattutto a tutti coloro che hanno interesse a portare idee innovative ed esperienza

Si ritiene positivo fornire e sollecitare contenuti che facciano luce su un fenomeno caratterizzato da nuovi comportamenti di addiction di massa, in una situazione economica difficile e che spinge a rivedere modelli di assistenza e di cura.

Ed è in questo senso che va il cambiamento di Mission, adeguandosi a criteri scientifici di accreditamento internazionale e rivolgendosi, sempre più, non solo a chi si occupa di dipendenze a livello professionale ma anche al mondo accademico.

L'obiettivo che ci si propone è presentare le più aggiornate e solide evidenze scientifiche, con un occhio attento e concreto alla possibilità di trasferirne l'azione sul territorio, guardando alle criticità dell'oggi ma pensando altresì ai mutamenti che inevitabilmente si produrranno nel fare prevenzione, cura e ricerca.

Guardare avanti e diffondere cultura, per contribuire al dibattito pubblico e influenzare le scelte dei decisori nella direzione della costruzione di modelli operativi appropriati, per il beneficio degli utenti, delle loro famiglie e delI'intera collettività.

Un grande sforzo per FeDerSerD, che potrà concretizzarsi con l'impegno di tutti! Tecnicamente, come potete vedere le modifiche riguardano per ora solo una parte della rivista, ripensata e ridisegnata nella sua struttura, prevedendo per alcuni contributi una procedura di referaggio doppiamente cieco (double blind peer review process), con revisori scelti in base a criteri di competenza e professionalità per garantire il livello qualitativo, la pertinenza e il rigore scientifico degli articoli accettati e pubblicati.

Per i dettagli della nuova procedura di sottomissione dei contributi scientifici a Mission, si rimanda alla pagina web visibile in Open Journal Systems www.francoangeli.it/riviste/sommario.asp?idRivista $=197$ \&lingua $=i t \& a n n o=2$ 016, la piattaforma online di FrancoAngeli per la gestione dei periodici e la pubblicazione dei contenuti in modalità open access.

Per ulteriori informazioni: www.federserd.it/index.cfm/Archivio-mission/ ?fuseaction=mission.

Oppure inviare una comunicazione email a missionredazione@gmail.com. Il resto del periodico segue le usuali modalità di sottomissione degli articoli.

Alfio Lucchini

Direttore scientifico

\section{FeDer5erD}

aderente a:
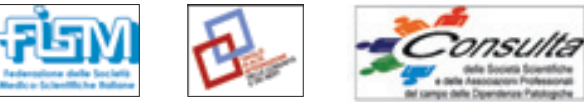

Sede legale: Via Giotto 3, 20144 Milano

\section{Direttivo Nazionale}

Pietro Fausto D'Egidio (presidente)

Roberta Balestra (vicepresidente)

Guido Faillace (segretario esecutivo)

Alessandro Coacci e Alfio Lucchini (past president)

Giancarlo Ardissone, Luigi Bartoletti,

Rosalba Cicalò, Edoardo Cozzolino,

Francesco De Matteis, Massimo Diana,

Donato Donnoli, Giuseppe Faro, Mara Gilioni,

Maria Luisa Grech, Debora Lageder,

Fernanda Magnelli, Antonella Manfredi,

Nicola Marrollo, Felice Nava, Marco Riglietta,

Gianna Sacchini, Giorgio Serio, Paola Trotta,

Concettina Varango, Franco Zuin,

Margherita Taddeo

Comitato Scientifico Nazionale

Felice Nava (direttore)

Massimo Diana e Marco Riglietta (vicedirettori)

\section{RICHIESTA DI ISCRIZIONE IN QUALITÀ DI "SOCIO ORDINARIO"}

Il versamento della quota associativa, pari a $€ \mathbf{5 0 , 0 0}$ per i laureati e a $€ \mathbf{3 0 , 0 0}$ per i non laureati, si può effettuare tramite:

$\square$ versamento sul Conto Corrente Bancario n. 000003417x16 intestato a FeDerSerD presso la Banca Popolare di Sondrio - Agenzia di Carimate - CIN D ABI 05696 CAB 51090

$\square$ versamento diretto ai referenti regionali con rilascio di ricevuta

L'accoglimento dell'istanza di iscrizione a FeDerSerD in qualità di Socio Ordinario avverrà nella prima riunione in calendario del Consiglio Direttivo e ne verrà data comunicazione e conferma con il rilascio e l'invio della Tessera Annuale.

ANNO 2018 - RICHIESTA DI ISCRIZIONE IN QUALITÀ DI "SOCIO ORDINARIO"

Da trasmettere per posta a Expo Point - via Matteotti, 3 - Mariano Comense (Co) o tramite fax al numero $031 / 751525$ o per e-mail federserd@expopoint.it

COGNOME

NOME

NATO A

INDIRIZZO (personale) $\quad$ PROVINCIA _ _ CAP

FAX

E-MAIL (per invio news e comunicazioni)

TITOLO DI STUDIO

DATA CONSEGUIMENTO TITOLO DI STUDIO

SPECIALIZZAZIONE

POSIZIONE PROFESSIONALE ATTUALE

INDIRIZZO (lavorativo)

TEL. FAX GELL. C-MAIL

PUBBLICAZIONI / ESPERIENZE CURRICOLARI DA SEGNALARE / PROGETTI SCIENTIFICI IN CORSO / AREE DI INTERESSE SCIENTIFICO / INTERESSI CULTURALI

Chiedo

$\square$ Di essere iscritto in qualità di SOCIO ORDINARIO per l'anno 2018

$\square$ Di rinnovare l'iscrizione in qualità di SOCIO ORDINARIO per l'anno 2018

a FeDerSerD - Federazione degli Operatori dei Dipartimenti e Servizi delle Dipendenze lì / / Firma

Si autorizzano FeDerSerD e la Segreteria Expopoint al trattamento dei dati inclusi nella presente scheda

Versamento quota associativa di $\square € \mathbf{5 0 , 0 0}$ (laureati) $\square € \mathbf{3 0 , 0 0}$ (non laureati)

(allegare copia bonifico bancario o ricevuta versamento)

Visto il Segretario Esecutivo Nazionale

Visto per approvazione: Il Presidente

Poste Italiane Spa - Sped. in Abb. Post. - D.L. 353/2003 (conv. in L. 27/02/2004 n. 46) art. 1, comma 1 - DCB Milano - Franco Angeli srl, viale Monza 106, 20127 Milano In caso di mancato recapito inviare a CMP Roserio per la restituzione al mittente previo pagamento resi. 